Para enlazar con este artículo / To link to this article:

http://dx.doi.org/10.6035/MonTI.2019.ne5.5

Para citar este artículo / To cite this article:

Galanes-Santos, Iolanda. (2019) "El redactor periodístico ante el neologismo. Estrategias comunicativas para reexpresar los conceptos de la crisis económica mundial." En: Montero Küpper, Silvia; Montserrat Vázquez Gestal \& Iván Puentes Rivera (eds.) 2019. Comunicación, Traducción e Interpretación / Communication, Translation and Interpreting. MonTI Special Issue 5, pp. 121-145.

\title{
EL REDACTOR PERIODÍSTICO ANTE EL NEOLOGISMO. ESTRATEGIAS COMUNICATIVAS PARA REEXPRESAR LOS CONCEPTOS DE LA CRISIS ECONÓMICA MUNDIAL ${ }^{1}$
}

\author{
EDITORS FACING NEOLOGISMS IN JOURNALISM. \\ COMMUNICATION STRATEGIES USED TO RESTATE THE \\ CONCEPTS OF THE GLOBAL ECONOMIC CRISIS
}

\author{
IOLANDA GALANES-SANTOS \\ iolag@uvigo.es \\ Universidad de Vigo
}

\section{Resumen}

El redactor, situado en la primera línea de las innovaciones comunicativas, incluye en sus artículos neologismos terminológicos para denominar los nuevos conceptos de la actualidad. La crisis económica mundial ha producido una constante renovación terminológica en el discurso periodístico. Las estrategias que el redactor utiliza para explicar un concepto nuevo dejan marcas discursivas en el texto (Hermans \& Vansteelandt 1999, Roldán 2012, Alves 2011 o Galanes 2017b). Estos marcadores permiten identificar las traducciones y las reexpresiones de neologismos terminológicos. Estos pueden ser préstamos, equivalencias traduccionales o bien reexpresiones que incorporan valores de la cultura meta, connotaciones o eufemismos. La selección de una u otra forma está condicionada por la estrategia comunicativa del redactor.

1. Miembro del Grupo de investigación en Estudos Literarios e Culturais, Tradución e Interpretación BiFeGa, financiado por la Xunta de Galicia: Programa de Consolidación e Estruturación de Unidades de Investigación Competitivas (Ref. ED431B 2017176). El 
En este artículo elaboramos una relación de marcadores discursivos de reexpresión de neologismos y analizamos su eficacia, también visibilizamos la intencionalidad del redactor cuando selecciona formas neológicas en el exhaustivo corpus de prensa DiCEM (2007-2017).

Palabras clave: Comunicación; Neología; Traducción; Marcadores; Prensa escrita

\begin{abstract}
Editors, located at the front line of communicative innovation, use terminological neologisms in their articles to designate the new concepts of today. The global economic crisis has brought about a constant terminological renovation within the journalistic discourse. The strategies that editors use to explain new concepts leave discursive markers in the text (Hermans \& Vansteelandt 1999, Roldán 2012, Alves 2011, López-Rodríguez 2017 or Galanes 2017b) that enable to identify translations and restatements of terminological neologisms. These may be loanwords, translational equivalences or restatements that incorporate values from the target culture, connotations or euphemisms. Choosing one form or another is conditioned by each editor's communicative strategy. In this article, we draft a list of discursive markers that restate neologisms, and we approach their typology according to the editors' intentions and strategies by researching into the exhaustive press corpus DiCEM (2007-2017).
\end{abstract}

Keywords: Communication; Neology; Translation; Discursive markers; Printed press

\title{
1. Introducción
}

La crisis económica mundial, desarrollada a partir de 2007, ha estado muy presente en los medios de comunicación. El debate sobre sus causas, los efectos en los sectores productivos y en la sociedad, así como sus variadas

grupo pertenece a la Rede Internacional de Investigación de Xestión da Comunicación (Ref. ED341D R2016/019 XESCOM), financiada por la Xunta de Galicia. El presente trabajo se ha realizado parcialmente durante su estancia de investigación en el Observatoire linguistique Sens-Texte de la Universidad de Montreal. Esta estancia ha sido financiada por el Ministerio de Educación, Cultura y Deporte en el marco del Programa Estatal de Promoción del Talento y su Empleabilidad en I+D+i, Subprograma Estatal de Movilidad, del Plan Estatal de I+D+I, en virtud de la Resolución de 11 de diciembre de 2017, de la Secretaría de Estado de Educación, Formación Profesional y Universidades [BOE de 18 de diciembre de 2017]. 
consecuencias consolidaron un discurso periodístico sobre la crisis ${ }^{2}$ en la última década. Es un evento considerado momento discursivo (Moirand 2007: 4) que, frente al instante, ha dado lugar a abundante producción mediática y ha dejado huella en discursos posteriores. Al mismo tiempo, se trata de un evento complejo con fases y manifestaciones singulares, también en el plano lingüístico (Galanes 2016).

En sus tareas cotidianas el comunicador se ve obligado a traducir, trasvasar culturalmente y divulgar conceptos especializados ignotos para el público meta de los medios de comunicación. Su condición de mediador, no necesariamente especialista en la materia, le obliga a documentarse de modo eficaz y urgente sobre la actualidad para poder comprender y trasmitir fielmente lo que está pasando en cada momento, que suele ser novedoso. Asistimos, por ejemplo, a la emergencia de nuevos conceptos (refundación del capitalismo Luna 2016), de nuevos términos (hipotecas de alto riesgo) y de nuevas metáforas terminológicas (hipoteca basura, hipoteca de baja calidad, hipoteca loca, etc.); surgen nuevos significados (para austeridad, indignado/a o rescate) y se vulgarizan conceptos especializados (prima de riesgo o activo Fernández 2016), etc. Todos ellos se registran primeramente en prensa y, por su novedad, han tenido que ser explicados por el redactor.

Se trata en palabras de Hernández Guerrero (2011: 104) de "una tarea compleja que lleva al redactor a seleccionar, filtrar, reorganizar, valorar, jerarquizar, distribuir y, también, traducir las informaciones". Esta mediación entre especialista y lego implica una operación interlingüística e intercultural que queda oculta bajo la consideración del artículo de prensa como producción original. La reescritura, en todas sus modalidades, es la causa de la invisibilidad de la traducción periodística (Hernández Guerrero 2011, Valdeón 2016, entre otros), que no está exenta de tensiones entre la extranjerización y la domesticación de contenidos (Bielsa 2016: 27 y ss.) y términos.

2. Sirvan de muestra los datos que nos ofrece Chavero (2015: 92 y ss.) en su análisis del discurso mediático de la legislatura 2008-2011 en España. Nos indica que entre septiembre de 2008 y diciembre 2009 los temas económicos representaron el 36\% de la agenda de los medios. La reforma laboral que se deriva de ella supuso en $37,4 \%$ de la agenda entre abril y junio de 2010. Y, por último, la ruptura de diálogo social hace que la crisis ocupe el 43,1\% de la agenda del primer trimestre de 2011. 
En el presente trabajo, a través del estudio de las estrategias de inserción de terminología especializada neológica en textos periodísticos, visibilizamos la operación implícita de traducción (y de divulgación) y estudiamos los elementos que son objeto de reformulación, al tiempo que sus soluciones.

Entre nuestros objetivos figuran el de crear una lista de marcadores lingüísticos de los "nuevos conceptos" divulgados a través de la prensa escrita en español (que denominamos MRNE) y también el de avanzar en la descripción y funcionalidad de las estrategias de mediación periodística. Esta lista de marcadores clasificada permitirá a terminólogos y traductores detectar operaciones traductivas o de divulgación en cualquier texto de prensa e identificar fácilmente los neologismos, sus componentes (denominación, concepto -intensión y extensión- referencia, etc.) y las soluciones adoptadas. Además, estos marcadores evidencian la intención comunicativa de cada denominación y señalan los elementos susceptibles de reformulación.

\section{Marco teórico}

De acuerdo con Hernández Guerrero (2011) existe una fuerte interacción entre Traducción y Periodismo en la prensa escrita española actual. Esta interacción no siempre presenta abiertamente los datos de traducción (título del original, nombre del traductor, etc.), sino que subyace bajo la forma de creación periodística. Ello convierte al periodista en redactor, pero también en traductor, adaptador, divulgador y mediador cultural. Asume múltiples tareas en un proceso que algunos denominan transediting (transedición), término acuñado por Stetting 1989 y definido como "as a new term to account for the fuzzy borderline between translating and editing" (Schäffner 2012). Otros autores, como Cheesman \& Nohl (2011) apud Valdeón (2016: 38), prefieren utilizar gatekeeping para referirse a estas transformaciones. La diferencia estriba en que la transedición se describe como "discursive re-intonations" e implica una mayor adaptación a la cultura meta, mientras que en el gatekeeping prevalece la selección previa al proceso de traducción.

Valdeón (2016: 39) indica que la traducción de noticias y otros textos periodísticos puede presentar diversas modalidades que van desde la traducción en sentido estricto a la reescritura. Ello deja margen para transformaciones lingüísticas, culturales e ideológicas, a las que precede una decisión o selección. La función seleccionadora (o gatekeeping) se opera por lo menos 
en dos instancias: los medios de comunicación en que se publica el artículo (normas editoriales) y el profesional de la comunicación (con sus propias normas).

De acuerdo con nuestros objetivos de estudiar los aspectos lingüísticos y comunicativos de la traducción periodística, analizamos la selección terminológica que realiza el redactor cuando recrea e informa sobre una realidad percibida como foránea o extraña para el público lector. Tomamos como objeto de estudio un tema de actualidad -la crisis económica mundial, surgida en Estados Unidos- cuya terminología ha sido, explícita o implícitamente traducida en las redacciones periodísticas.

No son pocos los trabajos del campo de la Terminología que analizan el discurso periodístico para detectar neologismos (o nuevos términos), como la red de Observatorios de neología NEOROM (Cabré 2006). La inmediatez de la transmisión de información global en las redacciones obliga al comunicador a acuñar urgentemente soluciones léxicas y terminológicas sobre temáticas desconocidas para el lector e, inicialmente, también para él mismo. Estos términos tienen, además, el valor de la espontaneidad por difundirse sin haber sido aún objeto de normalización terminológica (estandarización) por parte de las academias o de las autoridades lingüísticas pertinentes y, por supuesto, antes de su inclusión en diccionario.

En trabajos anteriores sobre la neología terminológica de la crisis económica (Galanes 2016, Galanes 2017a, Fernández \& Galanes 2018), nos hemos interesado por los neologismos y su variación denominativa, semántica y conceptual. Es frecuente que un concepto nuevo reciba en prensa más de una denominación y la selección de una determinada forma de referencia, frente a otras, está condicionada por la cultura meta, las editoras y la estrategia de comunicación del redactor. Así, por ejemplo, demostramos que la perspectiva comunicativa es relevante en la selección de las variantes terminológicas que designan a los créditos subprime (Fernández \& Galanes 2015: 271 y 278). De modo que la denominación original subprime mortgage loan se basa en la clasificación de la solvencia del prestatario (como parte de la escala credits subprime, credits Alt A, credits jumbos, credits (super) prime), mientras que en las lenguas romances predominan las denominaciones creadas desde la perspectiva del producto y no del individuo que lo contrata (hipoteca basura en español, crédit à risque en francés, titolo spazzatura en 
italiano o crédito malparado en portugués). Se evidencia así un cambio en la perspectiva comunicativa entre la denominación original y sus equivalencias en las otras culturas.

Nuestro trabajo sistemático en la elaboración del Diccionario de la Crisis Económica Mundial (DiCEM), nos ha enfrentado a multiplicidad de variantes para cada entrada, es decir, con la variación terminológica (de acuerdo con la Teoría Comunicativa de la Terminología de base descriptivista, Cabré 1999). Más allá de cada caso, nos hemos preguntado, a modo de hipótesis, cómo presenta el comunicador esas variantes denominativas y si existe en el discurso periodístico alguna marca de que se trata de un término "traducido" o trasvasado de otra cultura o ámbito especializado.

De modo experimental en Galanes (2017b), seleccionamos del corpus DiCEM con criterios cuantitativos un subcorpus de 27 anglicismos (subprime, stress test, credit default swap, core tier, quantitative easing, etc.). Analizamos su frecuencia y su cronología en 2 medios generales (El País y El Mundo) y 1 medio semiespecializado (Expansión) y llegamos a la conclusión de que estos términos en inglés son mucho más frecuentes y se introducen antes en la prensa semiespecializada, que en los medios de información general. Dado que el anglicismo convive en muchos textos con las formas nacionales, pretendemos sistematizar cómo se presentan ambas formas en texto. Para ello, utilizamos los estudios de neología traductiva de Hermans \& Vansteelandt (1999), Alves (2011) y Galanes (2017b: 133-135).

Otros trabajos abordan el estudio de la prensa y la intención comunicativa sobre fuentes estables (denominación de Hernández Guerrero 2011: 109), es decir, textos periodísticos firmados. Así, Orts (2017) identifica los marcadores textuales o interactivos y los interpersonales o interaccionales, todos ellos de tipo metadiscursivo, en textos autoriales de opinión. Por su parte, LópezRodríguez (2017) estudia los marcadores léxicos metalingüísticos explícitos (ELMO), que señalan la presencia de términos, en varios textos ordenados de mayor a menor grado de abstracción. Una de sus conclusiones es que los ELMO son más frecuentes cuanto más divulgativo sea el texto y pone como ejemplo la revista Muy Interesante (López-Rodríguez 2017: 157), frente a textos de mayor abstracción.

La documentación conjunta de anglicismos junto a sus equivalencias en español en prensa son una evidencia de traducción y su estudio nos sitúa 
necesariamente ante la perspectiva del comunicador. En el presente trabajo estudiamos la acción del redactor cuando reformula la información. Ello nos conduce al estudio de los marcadores de reformulación y al de sus estrategias, por lo que nos hemos propuesto como objetivos: 1. identificar y sistematizar los marcadores lingüísticos de reexpresión de neologismos en el total del corpus; y 2. analizar la estrategia de mediación y selección (comprensión, síntesis, traducción, mediación cultural, explicación, adaptación al destinario, adaptación al medio, etc.) en la reformulación. Se trata de estudiar las transformaciones (o manipulaciones Hernández Guerrero 2011: 109) realizadas en la localización de la noticia cuando el comunicador reformula su terminología neológica

La recopilación de marcas y marcadores de estudios previos, y su puesta a prueba en nuestro corpus, nos ha permitido elaborar una completa relación de marcadores lingüísticos, que denominamos marcadores de reformulación de neología especializada (MRNE). A partir de los ejemplos extraidos, ahondamos en la descripción de la mediación, identificando qué elementos son objeto de ella y las estrategias comunicativas en su reformulación. Con esa información optimizamos la lista de marcadores, clasificándolos según su ámbito de incidencia (la denominación, la intensión o la extensión del concepto, su definición, su explicación, etc.). Esta clasificación, además de novedosa, redunda en la futura utilidad de la citada lista, pues permite discriminar distintas soluciones para los diferentes problemas terminológicos de la traducción (denominativos, conceptuales etc.).

\section{Corpus}

Para el trabajo de campo utilizamos un corpus de prensa general y especializada, constituido como fuente de extracción del Diccionario de la Crisis Económica Mundial (DiCEM). Seleccionamos el subcorpus español, que recoge las noticias de prensa sobre crisis económica (entendida de modo integral como crisis económica, financiera, bancaria, hipotecaria, crediticia, de deuda, de la economía real y crisis política) aparecidas un día a la semana (los viernes) en 3 medios españoles (El País, El Mundo y Expansión) entre agosto de 2007 y diciembre de 2013. Para su actualización incluimos además los ejemplares de los viernes de Expansión de 2014 a 2017 y algunas calas de ejemplares de El Mundo y El País del mismo período y día. 


\begin{tabular}{|l|c|c|c|}
\hline & ejemplares & $\begin{array}{c}\mathbf{n} .^{\circ} \text { palabras } \\
\text { (tokens) }\end{array}$ & porcentaje \\
\hline El País (EP) & 448 & 2.736 .435 & $37,33 \%$ \\
\hline El Mundo (EM) & 331 & 1.538 .443 & $20,98 \%$ \\
\hline Expansión (EXP) & 474 & 3.055 .376 & $41,68 \%$ \\
\hline TOTAL & 1.253 & 7.330 .254 & \\
\hline
\end{tabular}

Hemos obtenido un corpus DiCEM ampliado a partir del vaciado de 1.253 ejemplares de esos 3 medios en el período que va desde septiembre de 2007 a diciembre de 2017 en las proporciones indicadas. El volumen del corpus es de 7.330.254 palabras, de las que el 41,68\% pertenecen a Expansión. En la ampliación, decidimos mantener una proporción superior de corpus general (próxima al 60\%) que de semiespecializado, porque la reexpresión es menos frecuente en textos más especializados y, por lo tanto, presentan menos marcadores con esa función (López-Rodríguez 2017: 160).

\section{Metodología}

De acuerdo con el Diccionario de términos clave de ELE (en adelante, DTCELE), los marcadores del discurso son "unidades lingüísticas invariables cuya función es señalar ("marcar") la relación que se establece entre dos segmentos textuales". En concreto, nos interesan los marcadores discursivos reformuladores, definidos en la misma fuente como elementos que "presenta(n) el miembro del discurso en el que se encuentran como una nueva formulación de lo que se quiere decir con un miembro anterior". En nuestro caso, uno de los miembros será necesariamente un neologismo terminológico.

Consideramos neologismos terminológicos las unidades percibidas como nuevas por el público meta del texto en que se insertan. La novedad puede deberse a que se trate de un concepto foráneo (traducción extralingüística), a que sea un concepto técnico divulgado por primera vez en ese ámbito o a que sea producto de la evolución del significado de un término (traducción intralingüística). En los estudios neológicos Rondeau (1981) distingue entre néologie d'origine (NO) y néologie d'appoint (NA) ou de transfert, y otros investigadores como Sager (apud Humbley, 2015: 42) las denominan neología primaria y secundaria, o bien, neología de formación y de traducción: 
On peut distinguer deux types de néologie : celle où la formation d'un nouveau terme, dans une langue précise, accompagne la formation d'un nouveau concept et celle où le terme existe déjà dans une langue et où un nouveau terme est créé dans une autre langue. La situation typique dans laquelle se déroule le premier processus est la situation de travail (le laboratoire de recherche, la fabrication de nouveaux produits, etc.). Le contexte classique de la deuxième forme de néologie est la traduction (Hermans \& Vansteelandt 1999: 37).

Nos interesa, por lo tanto, estudiar la neología terminológica secundaria o neología terminológica traductiva, en la que se incluyen las denominaciones técnicas extranjeras o de reciente divulgación (traducción inter e intralingüística) y conocer cómo se introducen estas formas en el discurso periodístico.

Para acometer el primero de nuestros objetivos, crear una lista sistematizada de marcadores de reformulación de neología especializada (MRNE), acudimos a trabajos sobre neología traductiva (Hermans y Vansteelandt, 1999), sobre la identificación de marcas de ese tipo de neología (Auger 2010: 101; Alves 2011: 98, para el portugués de Brasil y Galanes 2017b: 134-135, para el español), a los que añadimos los patrones léxico-sintácticos para la detección de términos y neologismos (Roldán 2012: 58), así como los marcadores metalingüísticos ELMO (identificados en Rodríguez-Penagos 2004 y reutilizados por López-Rodríguez 2017), que sirven de puente entre el conocimiento especializado y el destinatario general, lector de prensa.

Los marcadores discursivos reformuladores han sido clasificados en DTCELE por Martín Zorraquino \& Portolés (1999) como: reformuladores explicativos (es decir, o sea, etc.), de rectificación (mejor dicho, más bien, etc.), de distanciamiento (en cualquier caso, de todos modos, etc.) y recapitulativos (en suma, en conclusión, etc.). Para nuestros objetivos solo nos interesan aquellos que tienen incidencia en ámbito terminológico y sobre las diversas facetas del término (denominación, concepto, referencia, etc.).

Utilizamos marcador tanto para designar las unidades lingüísticas como las marcas gráficas de reformulación (corchetes, paréntesis, comillas, etc.). Para la confección de nuestra lista de MRNE, partimos de los 35 patrones léxico-semánticos de neologismos y términos ${ }^{3}$ (Roldán 2012) y de las marcas gráficas de la neología (Auger 2010). Más que ofrecer una simple lista, nos ha

3. Si bien muchos podrían agruparse por ser variaciones (formas conjugadas o flexionadas). 
parecido adecuado agruparlos. Así, a partir de Galanes (2017b: 134-135) distinguimos marcas gráficas que tienen por finalidad introducir explicitaciones; marcadores metalingüísticos (próximos a los ELMO); marcadores referenciales, que presentan referentes; marcadores de traducción, que ofrecen una equivalencia y, por último 2 subclases que inciden en el ámbito de la intensión conceptual, bien porque explican el término, bien porque lo definen.

\begin{tabular}{|c|c|}
\hline $\begin{array}{l}\text { Tipo de marcador de } \\
\text { reformulación }\end{array}$ & marcas \\
\hline $\begin{array}{l}\text { Marca gráfica de } \\
\text { explicitación }\end{array}$ & $\begin{array}{l}\text { itálica, comillas simples o dobles, paréntesis y } \\
\text { corchetes }\end{array}$ \\
\hline Marcadores metalingüísticos & $\begin{array}{l}\text { conocidalo (como), lo que se conoce, que se conoce, } \\
\text { denominado/a, } \\
\text { el nombrado, que recibe el nombre } \\
\text { llamado/a, lo que se llama, lo que se podría llamar, lo que } \\
\text { se ha venido en llamar, lo que se ha dado en llamar, etc. } \\
\text { término usado, etc. } \\
\text { a partir de ahora, de ahora en adelante, etc. } \\
\text { en la jerga económica, técnicamente, para los } \\
\text { especialistas, en argot financiero, etc. } \\
\text { literalmente, en inglés }\end{array}$ \\
\hline $\begin{array}{l}\text { Marcadores referenciales o } \\
\text { de designación }\end{array}$ & $\begin{array}{l}\text { entre otros, similares, semejantes a, como, como son etc. } \\
\text { de los que se distinguen los siguientes tipos/clases, en } \\
\text { concreto, entre las que se encuentran } \\
\text { por ejemplo, } \\
\text { en primer lugar, en segundo, por último+ sustantivo } \\
\text { tanto+ sustantivo como + sustantivo }\end{array}$ \\
\hline $\begin{array}{l}\text { Marcadores de traducción o } \\
\text { de adaptación }\end{array}$ & guiones, paréntesis, comillas, conjunción $o$ \\
\hline Marcadores de explicación & $\begin{array}{l}\text { se trata de, que, que consiste en, es decir, esto es, o sea, } \\
\text { etc. } \\
\text { aposición precedida de, }\end{array}$ \\
\hline Marcadores de definición & $\begin{array}{l}\text { que es, definido como, que se define, que se considera } \\
\text { (como), lo que se entiende como/por, }\end{array}$ \\
\hline
\end{tabular}

Marcadores de reformulación de neología especializada (MRNE). Elaboración propia

Aplicamos esta clasificación a nuestro corpus de prensa con ayuda del programa que concordancias WordSmith 7.0. A partir de su funcionalidad Concord 
somos capaces de recuperar del corpus DiCEM ejemplos de todos los tipos de marcas y marcadores que analizamos a continuación, excepto la itálica.

Una vez singularizados los contextos, analizamos qué elementos son objeto de reformulación ( $\mathrm{l}^{\text {er }}$ miembro); cómo se produce esta en cuanto a la estructura gramatical y orden de los elementos; qué significado añade cada uno de los marcadores y cuál es la solución que se presenta $\left(2^{\circ}\right.$ miembro de la reformulación). En definitiva, pretendemos así, conocer qué transformaciones se operan en cada uno de los planos del término, así como la intención del redactor que reformula el neologismo terminológico.

\section{Análisis}

\subsection{Marcas gráficas de explicitación}

De las marcas gráficas de neologismo en texto indicadas (itálica, comillas simples o dobles, paréntesis y corchetes), excluimos de nuestro análisis las itálicas por no ser posible recuperarlas con WordSmith, que trabaja sobre textos sin formato.

Las comillas simples son menos frecuentes que las comillas dobles y funcionan, junto con la itálica, como marca de extranjerismo, aunque también puede representar los usos figurados. Las comillas son utilizadas por el redactor como marcas de explicitación de contenidos diversos, de modo que introducen énfasis o presentan un término técnico, como en (1) o son indicio de un uso figurado (2).

(1) Eso es lo que estudia el Banco de España, la forma en que los bancos saquen de sus balances todo el negocio inmobiliario y lo gestionen de forma "bien separada" de su negocio bancario y eso es lo que se conoce como "desconsolidación", ha insistido. <EXP_20/04/2012_EMPR_EFE $>^{4}$

(2) ... las declaraciones hechas por el ministro de Economía, Luis de Guindos, que confirmaban que los accionistas de Novagalicia Banco, entre los que se encuentra la caja, deberán asumir, mediante una 'operación acordeón', la recapitalización del banco con fondos públicos europeos. <EXP_21/12/2012_EMP_EFE2>

4. El subrayado es nuestro. Los ejemplos figuran, en la medida de lo posible, identificados con la fórmula <Medio_Fecha_Sección_Autor>, siendo EXP la abreviatura de Expansión, EM de El Mundo y EP de El País. 
Los paréntesis delimitan un espacio de relación entre redactor y lector, en que aquel introduce aclaraciones. En el caso de que se utilicen anglicismos, ofrece entre paréntesis su traducción (3). O bien incluye el anglicismo entre paréntesis (4) o informaciones metalingüísticas como una denominación popular (5) o una especificación (6). En ese espacio de relación también tienen cabida la oración en lengua original que se traduce en texto (7) e incluso la extensión del concepto (8) o su explicación (9).

(3) La junta aprobará el dividendo a cuenta de 2012, que se situará en torno a los 0,3 euros por acción, y que se pagará de acuerdo con el mecanismo de scrip dividend (efectivo o acciones). <EXP_22/03/2013 _ POR_FUENTES M.Á.>

(4) La Reserva Federal debe poner fin a la inscripción automática en la aplicación de costosos programas de descubierto y darle a los consumidores la opción de aceptación o rechazo (opt-in o opt-out).<EM_14/08/2009_ ECO_RIVERA LAURA 2>

(5) Antes de la reforma, el empresario tenía la opción de asumir, de hecho, la improcedencia del despido pagando la indemnización correspondiente (el llamado despido exprés) o correr el riesgo ante el juez de que la rescisión fuera declarada improcedente <EP_16/03/2012_24_ECO_MANUEL V. GÓMEZ Y JOSÉ YOLDI>

(6) La otra palanca para reducir costes de financiación para Popular será también el plan de liquidez del BCE (el denominado TLTRO), que consiste en la concesión de préstamos a muy bajo coste con la condición de que este dinero sea utilizado para aumentar el flujo de crédito a la economía, especialmente a las pymes. <EXP_03/10/2014_BANCA_ROBERTO CASADO>

(7) A cambio, España tuvo que plegarse a un MoU (Memorando de Entendimiento) con severas condiciones que se han ido cumpliendo a rajatabla hasta la fecha. Entre dichas condiciones está la creación de una Institución independiente del gobierno que supervise el cumplimiento de la política fiscal ("Spain is also requested to establish an independent fiscal institution to provide analysis, advice and monitor fiscal policy").<EXP_19/10/2012_ECO_SÁNCHEZ QUIÑONES JESÚS>

(8) Los tres primeros rescates (bancario, compra de bonos por BCE y línea preventiva de crédito) no solucionan los problemas, pero sí otorgan tiempo para acometer las políticas necesarias para llevar a cabo las reformas necesarias. <EXP_19/10/2012_ECO_SÁNCHEZ QUIÑONES JESÚS> 
(9) Las pérdidas se explican por la necesidad de elevar las provisiones para cumplir con los dos Reales Decretos de reestructuración del sistema financiero. En concreto, las cajas han tenido que realizar dotaciones por pérdidas de activos financieros (inversiones inmobiliarias) por 12.751 millones de euros y las de activos no financieros (activos adjudicados) hasta los 1.328 millones.<EXP_28/09/2012_EMP_M. R.>

Esa misma función de crear un espacio de explicitación se nos presenta en los fragmentos entre corchetes, que se utilizan para aclarar información (10) $\mathrm{o}$ añadir un sentido figurado (11).

(10) Por otra parte, la vicepresidenta ha explicado que "no se ha aprobado el instrumento para facilitar la financiación y la cobertura de vencimientos de las CCAA [los llamados 'hispabonos'lporque puede ser que requiera algún cambio normativo y esto puede llevar más tiempo".<EXP_08/06/2012_ECO_AMIGOT-B ROIG-M>

(11) En su intervención ante la Asociación de Bancos Cooperativos de Fráncfort, Trichet instó a la banca a volver a su rol tradicional de dar un servicio a la economía real... Acusó a las entidades de haberse centrado demasiado en una "especulación sin freno" y en el "juego financiero" [en el sentido de "apuesta"]. <EXP_16/10/2009_INV_EXPANSION. $\mathrm{COM}>$

Como se puede ver en los ejemplos citados hasta el momento, en cada uno de ellos pueden combinarse marcas gráficas junto a los marcadores, que analizamos a continuación por lo que reutilizaremos algunos ejemplos.

\subsection{Marcadores metalingüísticos}

Los marcadores metalingüísticos señalan explícitamente a las denominaciones especializadas. La necesidad de referirse a ellas se debe a que designan conceptos desconocidos para el público meta, bien por ser innovadores o por su alta especialización.

Estos marcadores se construyen, en términos generales, con los verbos y colocaciones con conocer, denominar, nombrar, llamar, recibir el nombre, etc. Su formulación se realiza con formas no personales de los citados verbos, bien en participio, bien en forma impersonal (conocida/o (como), lo que se conoce, que se conoce, denominado/a, el nombrado, llamado/a, lo que se llama, término usado, que recibe el nombre, etc). 
Es muy relevante el tiempo de enunciación del verbo, que acostumbra a enunciarse de modo impersonal (participio, verbo impersonal, etc.), como estrategia comunicativa de distanciamiento frente a la enunciación de los textos de opinión, normalmente en $1^{\text {a }}$ persona de singular o plural. Sirvan de ejemplo de introducción de un tecnicismo (1) de variantes (metafóricas o no) (12) y de extranjerismos (13):

(12) Barclays invertirá en la Sareb, conocida también como banco malo. Aunque, de momento, se desconoce cuál sería su aportación. $<$ EXP_07/12/2012_POR_EXPANSION.COM4>

(13) Los denominados pfandbriefe son el veneno que lastra a muchos grandes bancos alemanes, entre ellos el Hypo Real Estate, salvado sólo por la campana de las ayudas públicas y en proceso de nacionalización.<EP_08/05/2009_01_POR_GÓMEZ JUAN, PÉREZ CLAUDI>

El distanciamiento resulta aún más evidente en formulaciones como lo que se podría llamar, lo que se ha venido en llamar, lo que se ha dado en llamar, etc.

(14) Urgen medidas para apuntalar a las familias y evitar así que miles de personas sigan engrosando la lista de la exclusión social. Lo que se ha dado en llamar sobreendeudamiento es quizá la situación más alarmante y los desahucios por no poder pagar las hipotecas, la cara más visible. <EP_24/02/2012_10_POR_MORÁN CARMEN>

Estas reformulaciones pueden añadir, además, una explicitación de la marca de tecnicismo (15) con fórmulas como en la jerga económica, técnicamente, para los especialistas, etc. $\mathrm{O}$ bien introducen un equivalente traduccional o traducción literal (16), o se indica la procedencia lingüística con el marcador en inglés. Del mismo modo que en el caso anterior, registramos ejemplos con acumulación de marcadores (17)

(15) "El Consejo", dijo, "quiere evitar caer en la trampa de la liquidez", lo que en la jerga económica viene a suponer que por muy barato que sea endeudarse y gastarse ese dinero -es decir, por muy bajos que estén los tipos- la gente no pedirá créditos ni gastará por temor. Además, destacó que la semilla de la crisis actual es precisamente el bajo precio del dinero.<EP_16/01/2009_01_POR_PÉREZ CLAUDI-GÓMEZ JUAN>

(16) Este sistema se conoce como Kurzarbeit (trabajo corto, literalmente) en Alemania y Austria y, de hecho, se practica en España y en muchos otros países en el sector de la automoción, especialmente, bajo la forma de EREs de reducción. <EP_04/12/2009_XX_OPI_HERCE JOSE A.> 
(17) Esta inyección de liquidez es lo que se llama técnicamente en inglés quantitative easing, un aumento excepcionalmente brusco de la masa monetaria en circulación con el doble objetivo de facilitar el crédito a las empresas y evitar que la inflación se sitúe por debajo del objetivo del 2\%. <EP_06/03/2009_XX_ECO_OPPENHEIMER WALTER>

En definitiva, las referencias metalingüísticas introducen variantes diastráticas, tecnicismos, extranjerismos, a través de fórmulas verbales despersonalizadas o de sustantivaciones. El valor pragmático que se introduce es el distanciamiento del redactor con el relato, pues en la medida en que presenta el término con estos marcadores y de ese modo se sitúa fuera del discurso (como emisor), profundiza más en el distanciamiento.

\subsection{Marcadores referenciales o de designación}

Estos marcadores ponen en relación un término con un objeto o elemento al que se puede aplicar la denominación, es decir, actúan sobre la extensión del término. Los marcadores son variados, unos sirven de antesala a una enumeración (por ejemplo, como son, de los que se distinguen los siguientes tipos/clases, en primer lugar, por último, etc. véase 18); otros introducen una especificación (por ejemplo, en concreto, entre otros, etc. 20); y, por último, pueden presentar una comparación (similares a, semejantes, etc. 19). Todos estos marcadores van seguidos de términos, normalmente con categoría de sustantivo, que es la más numerosa.

(18) Según han indicado fuentes financieras, Catalunya Banc tiene emitidos un total de 1.710 millones de euros en productos híbridos, como son las participaciones preferentes y la deuda subordinada perpetua y con vencimiento. <EXP_22/03/2013_EMP_EXPANSION.COM/EFE>

(19) El Gobierno estable en este Real Decreto la creación de las sociedades inmobiliarias para que los bancos enanejen sus activos inmobiliarios y, en concreto, los activos inmobiliarios "físicos", es decir los activos adjudicados en los canjes de deuda o embargos. <EXP_11/05/2012 EMPR_ZULOAGA J.-ROMANI MICHELA>

(20) Los planes de reinversión del BCE son similares a los programas de QE de EEUU y Reino Unido, donde la rentabilidad de los bonos del Tesoro a largo plazo sigue siendo baja. <EXP_4/08/2017_ECO_FINANCIAL TIMES > 
En general estos marcadores, en la medida en que presentan objetos, resultan objetivos pues se limitan, en general, a ejemplificar. Además, los últimos presentan comparaciones y en ellas participa activamente el criterio del redactor. En todos ellos, su finalidad es didáctica, aunque en la comparación también puede aludirse a la intensión (o rasgos definitorios) del concepto (21). En otros casos, se utilizan como aproximación traslativa, como paráfrasis (22).

(21) La CAM fue la primera caja de ahorros en salir a Bolsa con la emisión de cuotas participativas en julio de 2008. Las cuotas participativas son similares a las acciones, pero no tienen derechos políticos, lo que hizo que esta caja las emitiese para capitalizarse.<EM_22/07/2011_POR_ MAILLO JUAN EMILIO>

(22) Por este motivo, tal y como se recoge dentro de la presentación del Tesoro al extranjero, no se descarta recurrir a instrumentos menos comunes de financiación como títulos ligados a la inflación o préstamos negociables similares a los pagarés (schuldschein loans) <EP_17/12/2010_XX_ECO_ROMERO ÁLVARO>

\subsection{Marcadores de traducción o de adaptación}

El repertorio de marcadores de traducción o de adaptación es mucho más reducido que los vistos anteriormente y se mezclan marcadores (como la conjunción o) con marcas como los paréntesis (ejs. 3, 4, 7 y 16), las comillas o los guiones.

Como ya vimos, entre paréntesis puede figurar la forma original en inglés o la equivalencia traduccional en español. Es especialmente productivo este recurso en combinación con marcadores como en inglés para citar siglas originales (165 ejemplos de un total de 210 casos introducidos con ese marcador). La fórmula es común a los tres periódicos y el semiespecializado registra el $40 \%$ de casos (85), en coherencia con la representatividad global de este medio en el corpus.

El recurso a los guiones como marca es menos frecuente que el paréntesis y presenta tanto la traducción como los referentes del término.

(23) Durante ese tiempo, los inspectores de la UE o de la "troika" -el BCE, la Comisión Europea y el Fondo Monetario Internacional - evaluarían si un país está cumpliendo con las condiciones de su programa de ayuda. $<$ EXP_05/10/2012_ECO_EXPANSIÓN.COM 
El recurso a la conjunción o como marcador sirve a distintas funcionalidades, como presentar la traducción (24), incluso si esta equivalencia es dinámica, esto es, no idéntica (ejs. 25 y 26). El orden del anglicismo y la equivalencia traduccional puede variar, como en (27).

(24) Se ha fijado un precio de 80 puntos básicos por encima del índice de referencia para las emisiones de renta fija o midswap.<EXP_29/09/2017_ ECO_A.S.S >

(25) La futura legislación elimina trabas para invertir en capital riesgo, en fondos de inversión libre o hedge funds, así como fondos inmobiliarios y de infraestructuras. Se facilita en particular la inversión en fondos de capital riesgo europeos y en fondos de emprendimiento social europeo. $<$ EX_01/12/2017_08_ECO_E. del Pozo>

(26) Las apuestas bajistas consisten en pedir prestadas acciones de un determinado valor para venderlas con la esperanza de que su precio caiga y poder comprarlas en un futuro a un precio más barato para devolvérselas a su dueño, apuntándose las correspondientes plusvalías. Este tipo de estrategia es realizada principalmente por los fondos de alto riesgo o hedge funds. <EP_17/02/2012_22_ECO_DAVID FERNÁNDEZ>

(27) Los bancos aprovecharon para sacar de sus balances los bonos basados en hipotecas. ¿Cómo? Creando 'conduits' o vehículos especiales de inversión, que son entidades vinculadas al banco pero jurídicamente independientes de él. .<EM_06/02/2009_ECO_PARDO PABLO>

La utilización del marcador o también puede vincular sinónimos (28), cohipónimos (29), introducir una notación técnica (BB) (30) o presentar una sigla, junto a su desarrollo y viceversa $(31,32)$.

(28) La nota BBB está solo dos escalones por encima del nivel especulativo o de bono basura. O, visto desde el máximo, la novena de las 10 dentro de lo que se considera grado de inversión, o deuda con cierta seguridad de cobro. España recupera uno de los dos escalones perdidos en la última rebaja de S\&P, en octubre de 2012. <EP_23/05/2014_ECO_ MIGUEL JIMÉNEZ>

(29) El número total de lanzamientos hipotecarios o desahucios practicados entre abril y junio de este año se redujo un 1,9\% respecto del mismo periodo del año anterior, en tanto que las ejecuciones de inmuebles iniciadas se redujeron un $17,4 \%$, al pasar de 17.414 a 14.380 . <EXP_14/10/2016_ECONOMÍA_EFE>

(30) En una entrevista con la emisora Business TV, aseguró que, en realidad, los "ratings implícitos" que se derivan de la prima de riesgo de la deuda 
española en el mercado corresponderían a una calificación por debajo de los niveles de inversión, esto es, en la categoría de bonos basura o BB, seis escalones por debajo del nivel actual.<EP_20/01/2012_20_ ECO_GONZÁLEZ ALICIA>

(31) La prima de riesgo de Portugal también se ha reducido hasta los 442 puntos básicos. Los seguros de impago de deuda (credit default swaps o CDS) de España se han reducido al cierre de la sesión a 220.000 dólares, muy por debajo de los 246.350 dólares de los italianos $<$ EXP_01/11/2013_POR>

(32) Esta cantidad será la que reembolsarán de manera anticipada 35 bancos de la eurozona de los 523 y los 800 que acudieron a la primera y a la segunda barra libre a tres años (LTRO o Long Term Refinancing Operation), respectivamente. <EXP_08/03/2013_POR_VERBOM.L.>

En definitiva, entendemos que con estas marcas se relacionan anglicismo y solución nacional pero también pueden representar otros vínculos, de modo que se le ofrece a lector información sobre los referentes o también información nocional, como los cohipónimos. También es una estrategia de divulgación de información técnica (notación), de variantes denominativas (desarrollo de siglas), de sinónimos, etc. Todo ello para adaptar el texto al destinatario, a través de un proceso de "tradución intralingüística", que subyace a toda divulgación.

\subsection{Marcadores de explicación}

Estos marcadores introducen información conceptual, a través de fórmulas como se trata de, que, consiste en, es decir, esto es, o sea, etc. Además de incluir información conceptual de tipo técnico, en un esfuerzo por divulgarla (33), pueden añadir información adicional o una interpretación aclaratoria (34) o explicitar un eufemismo (35), un uso figurado o metafórico (36). En otros casos, el redactor utiliza sinónimos (37) o comparaciones (38) para explicar el concepto especializado.

(33) Una permuta de cobertura por impago es el llamado credit default swap, CDS en inglés. Se trata de seguros frente al impago de instrumentos de crédito, como los bonos emitidos por los países. <EM_6/07/2012_ECO>

(34) la mejor opción es pedir ayuda a la eurozona agarrándose a este texto que le va a permitir negociar un rescate blanco. O sea, sin que pierda la soberanía económica y sin necesidad de que venga la Troika a España a decirnos qué impuestos hay que subir o cuántos funcionarios se 
tienen que ir a la calle. $<$ EM_08/06/2012_POR_HITAElena_GALLEGO JAVIER G.>

(35) El Consejo de Ministros ha acordado este viernes a propuesta del Ministro del Interior, Jorge Fernández Díaz, un plan de "reordenación" de escoltas, que consiste en un recorte de efectivos para adaptar la dotación a la nueva situación tras el anunció del cese definitivo de ETA y a los principios de austeridad en el marco de la actual situación económica. <EXP_20/01/2012_ECO_EUROPA PRESS>

(36) La teoría del bazuca consiste en que si guardas armamento de gran calibre en el bolsillo y el mercado lo sabe, nunca tendrás que usarlo. La eurozona decidió ayer dotarse de arsenal suficiente<EP_12/02/2010_ XX_ECO_PÉREZ CLAUDI/ANDREU MISSÉ-1>

(37) Todos sabemos que hay quienes invierten en posiciones largas $[0$ sea, al alzal y otros que apuestan en corto [es decir, a la baja, terreno favorito de los especuladores más agresivos <EP_03/12/2010_01_POR_PÉREZ CLAUDI>

(38) Bienvenido a las finanzas del siglo XXI. Y a los activos 'sintéticos', o $\underline{\text { sea, que tienen menos parecido con la realidad como La Guerra de }}$ las Galaxias con los viajes en avión. <EM_11/05/2012_ECO_PARDO $\mathrm{PABLO}>$

De todos ellos, el marcador que introduce una visión más subjetiva o interpretativa en su explicación es o sea, por ello, lo hemos documentado en mayor medida en textos de opinión. Por otro lado, es muy frecuente en la reproducción de la oralidad, a juzgar por la cantidad de contextos en que figura formulado en estilo directo.

\subsection{Marcadores de definición}

El redactor puede introducir información nocional bajo patrones lexicográficos o terminográficos, es decir, con forma de definición, en la medida en que presenta los principales rasgos distintivos que singularizan al concepto, en ocasiones frente a conceptos semejantes (39). Es posible, por lo tanto, que incluya información sobre su naturaleza, funciones, modo, objetivos, modalidades, componentes, etc. Se introduce con fórmulas del tipo: que es, definido como, que se define, que se considera (como), lo que se entiende como/por, además de la utilización de marcas gráficas como paréntesis, guiones, coma (40) o dos puntos. Esta práctica es común a medios generalistas y semiespecializados, si bien la definición puede ser contextual (41). 
(39) ...en 2012 se habían realizado 91.622 ejecuciones hipotecarias que son las tramitadas por los juzgados de primera instancia en las que se ha exigido en pago de deudas garantizadas por propiedades hipotecarias. De todas ellas, 70.257 acabaron en los juzgados como lanzamientos, que son los cambios en la propiedad de un inmueble, ya sea rústico o urbano, de empresas o de particulares. $<$ EP_12/04/2013_01_POR_DE BARRÓN ÍNIGO>

(40) Solo hasta finales de año, el Fondo tiene pendiente el vencimiento de unos 3.000 millones de euros. La cantidad sube hasta los 18.000 si se contempla todo lo que se entiende convencionalmente como deuda a corto plazo, la que vence en menos de tres años. <EP_17/08/2012_19_ ECO_EL PAÍS>

(41) En este sentido, señala que los precios están regresando en muchos mercados a su "valor justo", definido como el ratio medio a largo plazo del precio de la vivienda respecto a los ingresos disponibles y los alquileres. <EXP_17/08/2012_EMP_EUROPA-PRESS>

En otras ocasiones su función es precisamente etiquetar o calificar el objeto designado. La intención del redactor es clasificar y la estrategia es la cita del concepto genérico (42). El carácter dinámico del fenómeno neológico nos presenta estas clasificaciones de modo inestable (43).

(42) La medida más controvertida hasta ahora ha sido una resolución anunciada por el presidente del CSIC el mes pasado imponiendo una limitación de gasto a cada uno de los institutos, medida definida como corralito por muchos científicos, ya que se les impide utilizar fondos que ellos han captado para su investigación en proyectos competitivos estatales e internacionales, en contratos con empresas, por patentes, por donaciones, etcétera. <EP_09/08/2013_01_POR_ALICIA RIVERA>

(43) Este marco operativo debe incluir una definición de lo que se consideran "activos heredados". Este es un tema clave para España porque los bancos recapitalizados por el Fondo de Reestructuración Ordenada Bancaria (Frob) con dinero del MEDE podrían entrar en esta categoría <EXP_14/12/2012_POR_MIQUEL ROIG>

En definitiva, hemos podido documentar en corpus buena parte de las marcas y marcadores propuestos y su contraste nos ha permitido tanto ampliar la lista inicial de marcadores (Galanes 2017b), como conocer qué elementos son susceptibles de mediación y de qué modo se realiza esta. 


\section{Conclusiones}

Los recientes estudios sobre traducción periodística han puesto el foco sobre una modalidad tradicionalmente poco estudiada. El periodista, al igual que el traductor, realiza funciones de mediación, que no siempre se evidencian en el artículo de prensa. A partir del estudio de las innovaciones terminológicas introducidas por la crisis económica mundial, hemos ahondado en el concepto, el objeto, las estrategias y los resultados de esa mediación intra e interlingüística y cultural.

Los extranjerismos (o anglicismos en Economía) son claramente objeto de mediación por parte del redactor, que los traduce, explica o define. Junto a ellos, hemos descubierto que otras unidades necesitan también de la intervención del redactor, como los sistemas expertos de notación (BBB), las siglas (normalmente en inglés, CDS), los tecnicismos en proceso de divulgación (ejecuciones hipotecarias vs. lanzamientos), las formas que presentan usos figurados (activos `sintéticos`), algunos términos metafóricos (operación acordeón) y determinados eufemismos (plan de "reordenación" de efectivos).

La acción del redactor se hace patente con la utilización de Marcas y Marcadores de reformulación de neología especializada (MRNE) que implican la realización de operaciones de explicitación (como las comillas o la itálica); de referencia metalingüística (denominada, etc.); de referencia designativa (por ejemplo); de traducción o de adaptación (conjunción o); de explicación (consiste en) o de definición (definido como). A partir de la recopilación de algunos de los marcadores en trabajos previos y, sobre todo, con el contraste en un corpus de prensa exhaustivo, conseguimos presentar más de 50 marcadores, además de sus respectivas variantes. Esta lista puede tomarse como referencia para identificar neologismos y reformulaciones en cualquier tipo de corpus especializado en español.

La visibilidad del redactor se muestra en diferentes grados en cada una de las operaciones de mediación. En las marcas gráficas (como las comillas) simplemente nos muestra que debemos interpretar el segmento entrecomillado en clave figurada. En otras ocasiones, crea a través de paréntesis o corchetes un espacio de interacción con el lector, como si de una acotación teatral se tratase. Otros marcadores de reformulación, como técnicamente, sitúan al redactor en posición intermedia entre la comunidad científica y el 
lector. Este distanciamiento también se evidencia en los recursos morfológicos (formas verbales impersonales, sustantivaciones, etc.) en las que diluye su enunciación, aunque nos haga partícipes de su aprendizaje (conocido como).

El resultado de la mediación (o segundo miembro de la reformulación) es variado. Una búsqueda con marcadores puede recuperar explicaciones, especificaciones, definiciones, denominaciones, sinónimos, cohipónimos, hiperónimos, paráfrasis, explicitaciones (desarrollos de siglas, por ej.), interpretaciones y también traducciones literales, equivalentes traduccionales, etc. Por ello, es pertinente clasificar los marcadores de reformulación (MRNE) según el tipo de información que proporcionan como resultado y según actúen sobre la denominación (explicitación, metalingüísticas, de tradución), sobre la designación o sobre el concepto (explicación, definición).

A modo de resultado y para visibilizar el complejo proceso reformulación periodística ofrecemos el siguiente esquema:

\begin{tabular}{|c|c|c|}
\hline Elemento reformulado & Reformulación & $\begin{array}{l}\text { Ámbito de incidencia/ } \\
\text { resultado }\end{array}$ \\
\hline $\begin{array}{l}\text { Anglicismo } \\
\text { Término especializado } \\
\text { Notación técnica y siglas } \\
\text { Tecnicismo } \\
\text { Variante discursiva } \\
\text { Uso figurado (metáfora, } \\
\text { eufemismo) } \\
\text { Etc. }\end{array}$ & $\begin{array}{l}\text { Explicitación } \rightarrow \\
\text { Referencia } \\
\text { metalingüística } \rightarrow \\
\text { Designación } \rightarrow \\
\text { Traducción e adaptación } \rightarrow \\
\text { Explicación } \rightarrow \\
\text { Definición } \rightarrow\end{array}$ & $\begin{array}{l}\text { Término } \\
\text { Denominación (variantes) } \\
\text { Extensión (referentes) } \\
\text { Equivalentes } \\
\text { Intensión conceptual } \\
\text { Intensión conceptual }\end{array}$ \\
\hline
\end{tabular}

Elementos, estrategias y resultado de reformulación. Elaboración propia.

Aunque es posible que en texto figuren simultáneamente varias marcas y marcadores (por ejemplo, comillas y marca de definición) y algunos marcadores son comunes a dos categorías, nos ha parecido útil clasificarlos para discriminar el tipo de información que recuperan. La clasificación según su función y ámbito de actuación (denominación, concepto, etc.) permite optimizar la relación de marcadores elaborada (MRNE) pues identifica neologismos, pero también señala el tipo de reformulación y su resultado (información nocional, denominativa, referencial, etc.). Es una información valiosa que el traductor y el terminólogo necesitan conocer en su quehacer cotidiano. 


\section{Referencias bibliográficas}

ALVES, Ieda Maria. (2011) "Neologia tradutiva em textos de Economia." ReCIT 2, pp. 97-109.

AUGER, Pierre. (2010) "Néologicité et extraction néologique automatisée." En: Cabré, María Teresa et al. (eds.) 2010 Actes del I Congrés Internacional de Neologia. Barcelona: IULA, pp. 117-121.

BIELSA, Esperança. (2016) "La traducción en los medios de comunicación: una perspectiva cosmopolita." En: Martín Ruano, Rosario \& África Vidal Claramonte (eds.) 2016. Traducción, medios de comunicación, opinión pública. Granada: Comares, pp. 17-34.

CABRÉ, María Teresa. (1999) La terminología. Representación y comunicación. Una teoría de base comunicativa y otros artículos. Barcelona: Institut Universitari de Lingüística Aplicada, Universitat Pompeu Fabra.

CABRÉ, María Teresa. (2006) "NEOROM, réseau d'observatoires de la néologie des langues romanes." Neologica: revue internationale de néologie 1, pp. 115-118. Chavero, Palmira. (2015) Prensa y política en tiempos de crisis: estudio de la legislatura 2008-2011. Madrid: Centro de Investigaciones Sociológicas.

FERnÁndez RodrígueZ, Áurea. (2016) "Del concepto a la denominación en traducción económica. El caso de activo." Revista de Filología e Linguística Portuguesa 18:1, pp. 115-144. DOI: <http://dx.doi.org/10.11606/issn.21769419.v18ilp115-144>

FERNÁNDEZ RodRíGUEZ, Áurea \& Iolanda Galanes Santos. (2015) "La crise hypothécaire et ses dénominations." Babel International Journal of translation 61:2, pp. 265-281. DOI: <http://dx.doi.org/10.1075/babel.61.2.06fer>

FERNÁNDEZ RodríGUEZ, Áurea \& Iolanda Galanes Santos. (2018) "Neologisms, metaphors and new concepts for writing media news." En: Túñez-López, José Miguel; Valentín-Alejandro Martínez-Fernández; Xosé López-García; José Rúas-Araújo \& Francisco Campos-Freire (eds.) 2018. Communication: Innovation \& Quality. Springer.

GALANES SANTOS, Iolanda. (2016) "La crisis económica mundial: un concepto complejo con múltiples denominaciones." Revista de Filología e Lingüística Portuguesa 18:1, pp. 5-41. DOI: <http://dx.doi.org/10.11606/issn.2176-9419. v18ilp5-41 >

GALANES SAntos, Iolanda. (2017a) "Neologismos de la crisis: estudio contrastivo de sus denominaciones en la prensa gallega y española." En: Sánchez 
Ibáñez, Miguel et al. (eds.) 2017. La renovación léxica en las lenguas románicas. Proyectos y perspectivas. Murcia: Editum, pp. 91-105.

GALANES SANTOS, Iolanda. (2017b) "Neologismos conceptuales y referenciales: aportaciones desde la neología traductiva de la crisis." En: Vargas, Chelo \& Miguel Ángel Candel Mora (eds.) 2017. Temas actuales de terminología y estudios sobre el léxico. Granada: Comares, pp. 125-146.

Hermans, Adrien \& Andrée Vansteelandt. (1999) "Néologie traductive." Terminologies nouvelles 19, pp. 37-43.

HERNÁNDEZ GUERRERO, María José. (2011) "Presencia y utilización de la traducción en la prensa española." Meta 56:1, pp. 101-118. DOI : <http://dx.doi. org/10.7202/1003512ar >

HumbleY, John. (2015) "La néologie dans les langues de spécialité." En: Alves, Ieda Maria \& Eliane Simões Pereira (eds.) 2015. Neologia das línguas românicas. São Paulo: Humanitas, pp. 39-74.

LÓPEZ RodríGUEZ, Clara Inés. (2017) "Marcadores metalingüísticos explícitos y nivel de especialización: aplicaciones para la extracción de terminología." En: Chelo Vargas, Chelo \& Miguel Ángel Candel Mora (eds.) 2017. Temas actuales de terminología y estudios sobre el léxico. Granada: Comares, pp. 147-162.

Luna Alonso, Ana. (2016) "La `refundación del capitalismo’ Estudio terminológico en la prensa francesa y española." Revista de Filología e Linguística Portuguesa 18:1, pp. 145-174. DOI: <http://dx.doi.org/10.11606/issn.21769419.v18ilp145-174>

MARTín Zorraquino, María Antonia y José Portolés. (1999) "Los marcadores del discurso." En: Bosque, Ignacio \& Violeta Demonte (dirs.) 1999. Gramática descriptiva de la lengua española. Madrid: Espasa Calpe, pp. 4051-4213.

Molrand, Sophie. (2007) Les discours de la presse quotidienne. París: Presses Universitaires de France.

ORTS LLOPIS, María Ángeles. (2017) "Dos formas de contemplar la opinión periodística: Diferencias léxico-retóricas en las columnas económicas en inglés y en español." En: Vargas, Chelo \& Miguel Ángel Candel Mora (eds.) 2017. Temas actuales de terminología y estudios sobre el léxico. Granada: Comares, pp. 99-124.

ROLDÁN VENDREll, Mercedes. (2012) "Detección, análisis y clasificación de neologismos en corpus especializados: marcadores de creación léxica." En: Dury, Pascaline; José Carlos de Hoyos; Julie Makri-Morel; François Maniez; Vincent Renner \& María Belén Villar Díaz (dirs.) 2012. La néologie en langue 
de spécialité. Detection, implantation et circulation des nouveaux termes. Lyon: Centre de Recherche en Terminologie et Traduction, Université Lumière Lyon 2, pp. 51-74.

SCHÄFFNER, Christina. (2012) "Rethinking Transediting." Meta 57:4, pp. 866-883.

DOI: <http://dx.doi.org/10.7202/1021222ar>

VALDEÓN, Roberto A. (2016) "Traducción periodística y gatekeeping." En: Martín Ruano, Rosario \& África Vidal Claramonte (eds.) 2016. Traducción, medios de comunicación, opinión pública. Granada: Comares, pp. 35-51.

VARIOS AUtORES (s.d.) Diccionario de términos clave de ELE. Madrid: Centro Virtual Cervantes. Versión electrónica: < https://cvc.cervantes.es/ENSENANZA/ biblioteca_ele/diccio_ele/default.htm>

\section{NOTA BIOGRÁFICA / BIONOTE}

IOlAnda Galanes SAntos (Vigo, Spain) es licenciada y doctora en Filología Gallega y Portuguesa por la Universidade de Santiago de Compostela. Es profesora titular en el Departamento de Traducción y Lingüística de la Universidad de Vigo. Su investigación se enmarca en los Estudios de Traducción y la Terminología. Ha coordinado un proyecto ministerial de cooperación interuniversitaria junto con la Universidade de São Paulo sobre las imágenes de la crisis en la terminología de la prensa escrita.

Iolanda Galanes SAntos (Vigo, Spain) has a degree in Romance Philology and she is a Doctor Galician-Portuguese Philology by the University of Santiago de Compostela. She is currently senior lecturer at the Department of Translation and Linguistics at the University of Vigo. Her research areas devoted to Translation Studies and Terminology. She was leader in a university cooperation project with the University of São Paulo in order to study the imagery of the crisis through the terms used in the press. 